\title{
Intelligence and cognitive flexibility: Fluid intelligence correlates with feature "unbinding" across perception and action
}

\author{
LORENZA S. COLZATO, NELLEKE C. VAN WOUWE, TRISTAN J. LAVENDER, \\ and BERNHARD HOMMEL \\ Leiden University, Leiden, The Netherlands
}

\begin{abstract}
People integrate the features of perceived events and of action plans, as well as of episodic stimulusresponse relations, into event files. We investigated whether the management of event files, and particularly the speed of updating the binding between the task-relevant stimulus feature and the response, correlates with fluid intelligence. Indeed, the performance of participants scoring high on Raven's Standard Progressive Matrices test was less impaired by a mismatch between the stimulus-response relation in the current and the previous trial. This result suggests that high intelligence is accompanied by a higher degree of flexibility in handling event files-that is, by higher efficiency in updating episodic representations.
\end{abstract}

One of the most discussed issues in cognitive psychology is what has become known as the binding problem (Treisman, 1996) - that is, the question of how the human (or primate) brain is able to properly integrate all information about a particular event. In perception, there must be a mechanism that functionally links the features of an object to what Kahneman, Treisman, and Gibbs (1992) have called an object file - that is, a temporary cognitive structure containing all the perceptual information about an object, and perhaps even episodic and semantic information. Since comparable binding problems exist in action planning (Stoet \& Hommel, 1999) and in coordinating perceptual codes and action plans (Hommel, 1998; Hommel, Müsseler, Aschersleben, \& Prinz, 2001), it makes sense to assume that perceiving and acting require the creation of all sorts of event files (Hommel, 1998, 2004). In this article, we address the question of whether and how the cognitive management of event files is correlated with fluid intelligence, as measured by Spearman's $g$. There are three lines of reasoning that motivated us to consider a link between intelligence and event file handling.

First, event files are temporary structures that must be held and updated in working memory - a system whose flexibility has been shown to correlate with fluid intelligence (Duncan et al., 2000). To see how intelligence may relate to event files, let us consider the task from Hommel (1998), which we adopted for the present study (see Figure 1). In this task, participants are cued to prepare a left- or right-hand keypress (R1), which they carry out as soon as a first stimulus (S1) appears. The identity of S1

Correspondence relating to this article may be sent to L. S. Colzato, Leiden University, Department of Psychology, Cognitive Psychology Unit, Postbus 9555, 2300 RB Leiden, The Netherlands (e-mail: colzato@ fsw.leidenuniv.nl). does not matter for the response, but it varies in shape, location, and color. One second later a second stimulus (S2) appears, signaling Response 2 (R2), a binary-choice response to the shape of S2 (S2 color and location are entirely irrelevant to this version of the task). Performance in such a task reveals interesting interactions between repetition effects: Performance is impaired in partialrepetition trials - that is, if one of the stimulus features or the response is repeated but another element is not (e.g., if shape repeats but location does not, or vice versa, or shape repeats but the response does not, or vice versa). These partial-repetition costs suggest that the stimulus and response features of $\mathrm{S} 1$ and $\mathrm{R} 1$ are still bound when S2 appears, so that repeating a given feature (in S2) will retrieve all the event files the code of that feature has become a part of (Hommel, 1998, 2004). This creates conflict between the retrieved codes and those activated by the current $\mathbf{S} 2$, thus delaying reaction times and increasing error rates. These effects can be considered to represent the costs incurred by updating (i.e., modifying the structure of ) an event file, and thus to be a measure of the flexibility in managing one's cognitive representations. If so, and if we consider that working-memory-related measures of flexibility are associated with fluid intelligence measures (Duncan et al., 2000), we would expect that partial-repetition costs would be reduced in people scoring high in fluid intelligence. Since updating would only be necessary for the task-relevant feature relation (here, between stimulus shape and response), we would expect that the impact of intelligence is restricted to the corresponding (i.e., shape-response) binding.

Second, Hommel, Kray, and Lindenberger (2006) observed that children and older adults produce larger partial-repetition costs than young adults. Performance decrements in children are commonly attributed to the slow maturation of the frontal lobe (Diamond, 1990), the 


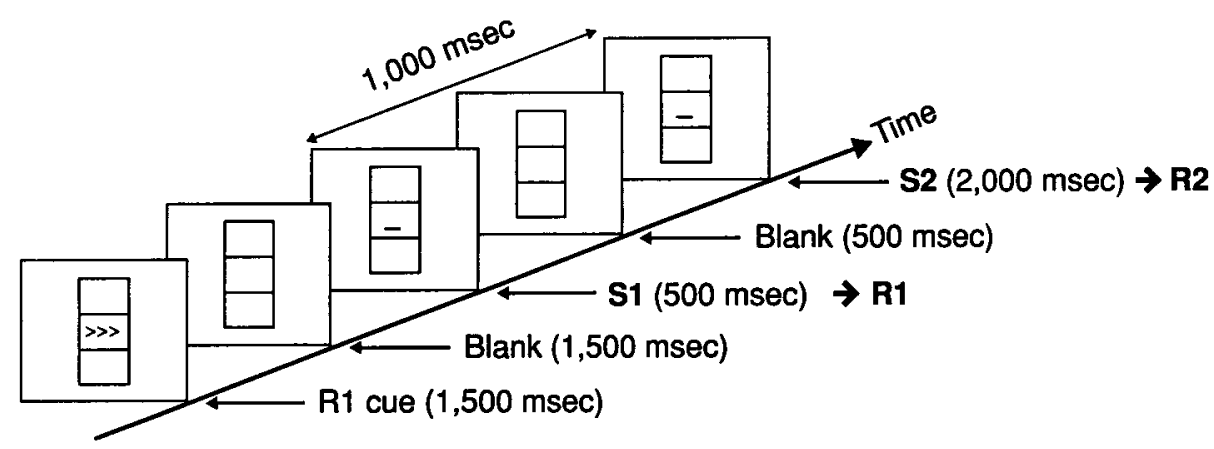

Figure 1. Sequence of events in the present experiments (see Hommel, 1998). A response cue signaled a left or right keypress (R1) that was to be delayed until presentation of the first stimulus (S1), a red or green, vertical or horizontal line in the top or bottom box. The second stimulus (S2) appeared $1 \mathrm{sec}$ later, and was another red or green, vertical or horizontal line in the top or bottom box. S2 shape signaled the second response (R2), also a speeded left or right keypress. $R 2$ speed and accuracy were analyzed as functions of the repetition versus alternation of stimulus shape, color, location, and response.

functioning of which is essential for performance in working memory tasks (Gathercole, 1999) and intelligence tests (Duncan et al., 2000). Likewise, decrements in old age have been linked to the particularly fast degeneration of the frontal lobe (Hasher \& Zacks, 1988), suggesting that increased partial-repetition costs may reflect frontallobe functioning. Along the lines of Duncan et al. (2000), this implies that high intelligence may facilitate the management of event files and, thus, reduce partial-repetition costs.

Third, it has been hypothesized that neural synchronization plays a key role in feature integration (Singer, 1994; Treisman, 1996). That is, cell populations that represent the features of a given object or action plan may synchronize their firing rates, so as to create a coherent neural structure. In support of this idea, we (Colzato, Erasmus, \& Hommel, 2004; Colzato, Fagioli, Erasmus, \& Hommel, 2005) recently demonstrated that partial-repetition costs are systematically modulated by drugs that can be expected to affect neural synchronization in the visual cortex (Rodriguez, Kallenbach, Singer, \& Munk, 2004). In view of evidence that higher intelligence is associated with stronger neural synchronization (Anokhin, Lutzenberger, \& Birbaumer, 1999), one may thus speculate that intelligence and the handling of event files are related.

To summarize, we investigated whether the level of fluid intelligence (low, average, or high) would correlate with our behavioral measure of event file management. In particular, we hypothesized that partial-repetition costs for the (most) task-relevant binding (shape with response) would be reduced for participants scoring high in fluid intelligence.

\section{METHOD}

\section{Partịcipants}

Forty-eight volunteers took part, for pay, in the study. All reported having normal or corrected-to-normal vision and were not familiar with the purpose of the experiment.

\section{Apparatus and Stimuli}

The experiment was controlled by a Targa Pentium III computer, attached to a Targa TM 1769-A 17-in. monitor. Participants faced three gray square outlines, vertically arranged, as illustrated in Figure 1. From a viewing distance of about $60 \mathrm{~cm}$, each of these frames measured $2.6^{\circ} \times 3.1^{\circ}$. A vertical line $\left(0.1^{\circ} \times 0.6^{\circ}\right)$ and a horizontal line $\left(0.3^{\circ} \times 0.1^{\circ}\right)$ served as $\mathrm{S} 1$ and $\mathrm{S} 2$ alternatives, which were presented in red or green in the top or bottom frame. Response cues were presented in the middle frame (see Figure 1), with rows of three left- or right-pointing arrows indicating a left or right keypress, respectively. Responses to S1 and S2 were made by pressing the left or right shift key of the computer keyboard with the corresponding index finger.

\section{Procedure and Design}

Individual IQs were determined by means of a 30-min reasoningbased intelligence test (Raven's Standard Progressive Matrices, or SPM). The SPM is a measure of Spearman's $g$ factor and is taken to implicate working memory capacity (Carpenter, Just, \& Shell, 1990). It measures an individual's ability to create perceptual relations and to reason by analogy, independent of language and formal schooling. Our sample did not cover the IQ range below 80 and is thus somewhat "positively biased." We formed three groups corresponding to the different IQ levels: relatively low (15 participants, 80-99 IQ), average to high (17 participants, 100-120 IQ), and very high (16 participants, 121-140 IQ).

The actual experiment consisted of a 50-min session in which participants completed a version of the task adopted from Hommel (1998; see Figure 1). Participants faced three gray, vertically arranged boxes in the middle of the monitor and carried out two responses per trial. $\mathrm{R} 1$ was a delayed simple reaction with the left or right key, as indicated by a $100 \%$-valid response cue (left- or rightpointing arrow in the middle box) that preceded the trigger stimulus S1 by $3,000 \mathrm{msec}$. S1 varied randomly in shape (a thin vertical or horizontal line), color (red or green), and location (top or bottom box). R1 was to be carried out as soon as S1 appeared, independent of its shape, color, or location; that is, participants were encouraged to respond to the mere onset of S1. R2 was a binary-choice reaction to the shape of S2 (vertical or horizontal orientation), a stimulus that also appeared in red or green, and in the top or bottom box, and was presented 1,000 msec after S1 onset. Each session was composed of a factorial combination of the two possible shapes, colors, and locations of $\mathrm{S} 2$ and the repetition versus alternation of shape, color, location, and response, with three replications per condition. 
Table 1

Means of Mean Reaction Times for Responses to Stimulus 2 (RTs, in Milliseconds) and Percentages of Error on Response 2 (PEs) as a Function of Intelligence (Low vs. Average vs. High), Match Between Responses 1 and 2, and Feature Match Between Stimulus 1 and Stimulus 2

\begin{tabular}{|c|c|c|c|c|c|c|c|c|c|c|c|c|}
\hline \multirow{3}{*}{$\begin{array}{c}\text { Stimulus } \\
\text { Feature(s) } \\
\text { Repeated } \\
\end{array}$} & \multicolumn{4}{|c|}{ Low IQ } & \multicolumn{4}{|c|}{ Average IQ } & \multicolumn{4}{|c|}{ High IQ } \\
\hline & \multicolumn{2}{|c|}{$\begin{array}{l}\text { Response } \\
\text { Repeated } \\
\end{array}$} & \multicolumn{2}{|c|}{$\begin{array}{l}\text { Response } \\
\text { Alternated }\end{array}$} & \multicolumn{2}{|c|}{$\begin{array}{l}\text { Response } \\
\text { Repeated }\end{array}$} & \multicolumn{2}{|c|}{$\begin{array}{c}\text { Response } \\
\text { Alternated }\end{array}$} & \multicolumn{2}{|c|}{$\begin{array}{l}\text { Response } \\
\text { Repeated }\end{array}$} & \multicolumn{2}{|c|}{$\begin{array}{l}\text { Response } \\
\text { Alternated }\end{array}$} \\
\hline & RT & $\mathrm{PF}$ & DT & PF & RT & PE & DT & $\mathrm{PE}$ & DT & $\mathrm{PF}$ & & PE \\
\hline & & & & & & & & & & & & \\
\hline & & & & & & & & & & & & \\
\hline & & & & & & & & & & & & 1 \\
\hline & & & & & & & & & & & & \\
\hline & & & & & & & & & & & & \\
\hline & & & & & & & & & & & & \\
\hline & & & & & & & & & & 16.4 & & \\
\hline$-\mathrm{C}+\mathrm{L}$ & 446 & 19.5 & 461 & 26.2 & 428 & 9.9 & 457 & 22.8 & 390 & 3.9 & 407 & 16.0 \\
\hline
\end{tabular}

\section{RESULTS}

After excluding trials with missing $(>1,500 \mathrm{msec})$ or anticipatory $(<200 \mathrm{msec})$ responses, mean reaction times (RTs) and proportions of error for R2 were analyzed (see Table 1 for means). ANOVAs were run with the repetition versus alternation of response $(\mathrm{R} 1 \rightarrow \mathrm{R} 2)$, stimulus shape, color, and location $(\mathrm{S} 1 \rightarrow \mathrm{S} 2)$ as within-participants factors and with IQ level (high vs. average vs. low) as a betweenparticipants factor. Replicating earlier findings (Hommel, 1998; Hommel \& Colzato, 2004), RTs revealed significant interactions between shape and location $[F(1,47)=16.55$, $p=.001]$, response and shape $[F(1,47)=52.82, p=.001]$, and response and location $[F(1,47)=8.59, p=.005]$ : Repeating one but not the other feature slowed down responding. IQ level impacted only the task-relevant binding of shape and response, thus producing a three-way interaction $[F(1,47)=5.12, p=.010]$. Figure 2 suggests that the shape $\times$ response interaction was reliable for all three IQ levels $[F(1,14)=25.82, p=.001 ; F(1,16)=11.48, p=$ .004 ; and $F(1,15)=5.94, p=.028$, for low, average, and high IQ, respectively]. However, the interaction was also much reduced in the high-IQ group. In other words, high IQ minimized partial-repetition costs, just as expected.

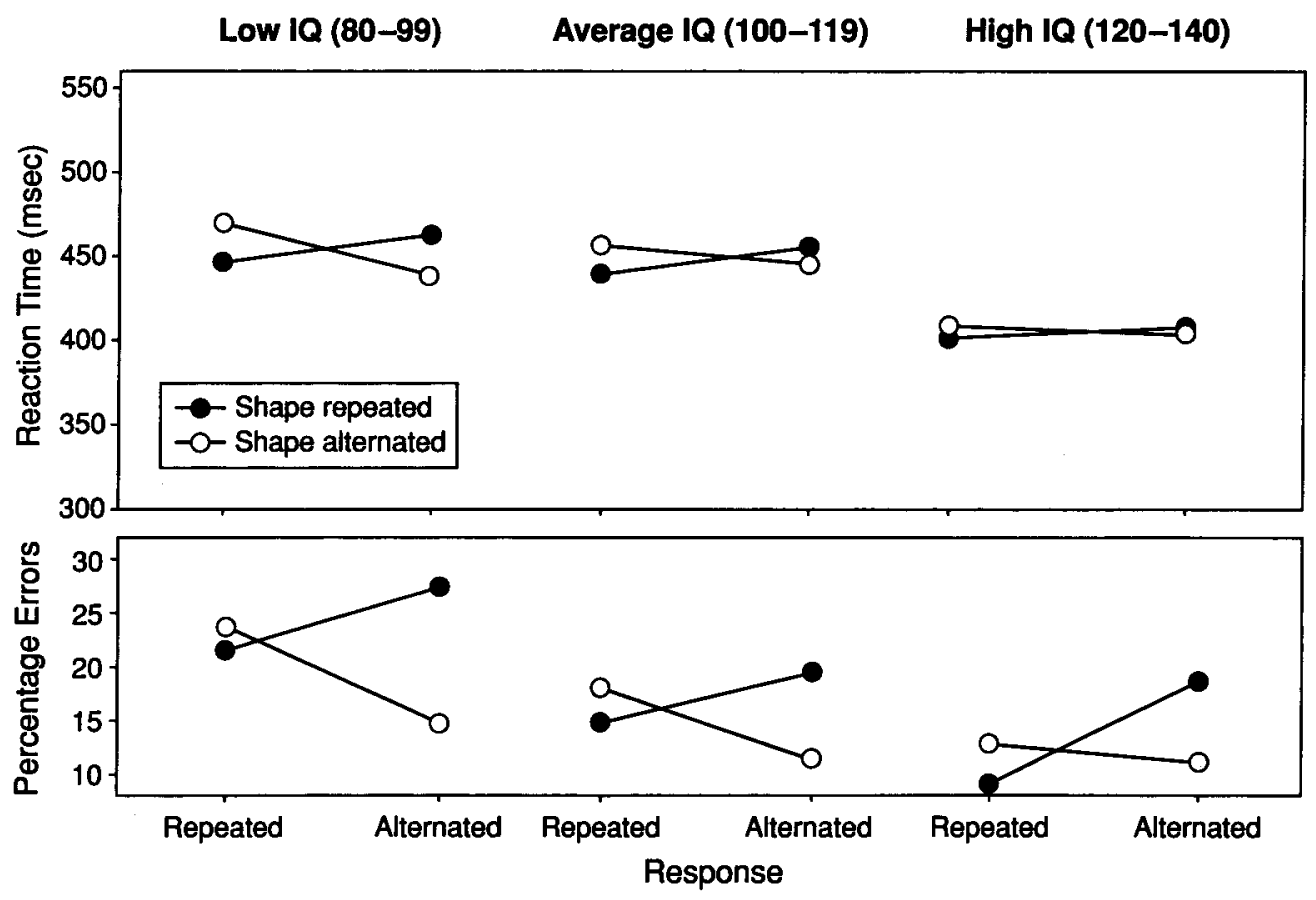

Figure 2. Mean reaction times and percentages of error as functions of IQ level (low vs. average vs. high) and the repetition versus alternation of response and stimulus shape. White dots on the left and black dots on the right side of each graph refer to a stimulus constellation that requires "unbinding"; that is, the stimulus shape or the response changes, while the other does not. 
The error rates followed the same pattern: Response produced a main effect $[F(1,47)=10.65, p=.001]$ and interacted with shape $[F(1,47)=46.47, p=.001]$, location $[F(1,47)=26.05, p=.001]$, and color $[F(1,47)=$ $16.01, p=.001]$. The interaction between shape and color also reached significance $[F(1,47)=5.31, p=.026]$. All four interactions were due to fewer errors in conditions in which both features were repeated or both alternated, as compared with conditions in which one feature but not the other was repeated. IQ level interacted only with shape (a task-relevant feature) $[F(1,47)=4.82, p=.012]$, such that repetition was an advantage only for the high-IQ group.

It is obvious from Figure 2 that the high-IQ group was faster than the other two groups. One may thus be tempted to consider that higher speed, not higher intelligence, was the reason why the high-IQ group showed reduced partialrepetition costs. With regard to interindividual differences in speed, this argument is true almost by definition and therefore not particularly interesting: RT speed is known to be a particularly reliable predictor of the level of fluid intelligence, and most intelligence models include speed as a component (see, e.g., Deary, 2000; Jensen, 1993; Vernon, 1987), which is reflected in the fact that many IQ tests test aspects of mental and/or sensorimotor speed. Indeed, in the present study, the individual size of partialoverlap costs associated with shape-response binding (mean RT for the two partial-overlap conditions minus mean RT for complete shape-response repetition and alternation) correlated highly with both IQ (as measured by the Raven score; $r^{2}=-.43, p=.002$ ) and RT level (as measured by the mean RT for R1; $r^{2}=.49, p<.001$ ), and IQ correlated with speed $\left(r^{2}=-.47, p=.001\right)$. A regression analysis with the shape-response partial-overlap costs as the dependent variable and individual IQ and response speed as predictors revealed that entering speed after IQ increased the explained variance significantly, from $16.7 \%$ to $25.4 \%\left[R_{\text {change }}^{2}=.10, F(1,45)=6.34, p<\right.$ $.01]$, but entering IQ after speed had only a marginal effect [from $23.4 \%$ to $28.5 \%$; $R_{\text {change }}^{2}=.05, F(1,45)=3.25, p<$
.08]. These observations support the assumption of a close (but not perfect) relationship between fluid IQ and RT.

More problematic for our argument would be a role of intraindividual differences in speed. If partial-overlap costs are tied to a particular (slow) RT range, the more intelligent participants might show less of a cost simply because their performance does not fall into this range as often as the performance of less intelligent participants does. If what truly counts is speed, independent of the IQ level of the given participant, this would speak against a direct connection between feature unbinding and intelligence. Fortunately, there are reasons to rule out this possibility. First, there is evidence that as such, a faster RT level does not necessarily reduce overlap costs. Recent drug studies have shown that caffeine consumption speeds up RTs and yet produces larger partial-overlap costs than in a control condition (Colzato et al., 2005), whereas alcohol consumption slows down performance and produces smaller costs (Colzato et al., 2004). Second, detailed analyses of the present data revealed that high-IQ participants showed smaller costs even in trials in which they were as slow as low-IQ participants. To test this finding, we Vincentized ${ }^{1}$ the RT data for each condition and participant (Ratcliff, 1979) so as to provide us with two RT means per condition: one for the fast and one for the slow half of the individual RT distribution. Even though the crucial three-way interaction of shape, response, and IQ was not reliably modified by relative response speed $(p>.3)$, separate analyses showed that the shape $\times$ response interaction was modified by response speed in the low-IQ group $[F(1,14)=10.27, p=.006]$, but not in the average-IQ $[F(1,16)=2.42, p=.14]$ or the highIQ $[F(1,15)<1]$ group. As is shown in Figure 3, slower responses were associated with larger overlap costs in the low-IQ group, whereas the costs were fairly independent of RT level in the other two groups. Thus, it is true that particularly fast responses may make it difficult to diagnose the presence of partial-overlap costs: Responses may simply be selected and carried out so quickly that they

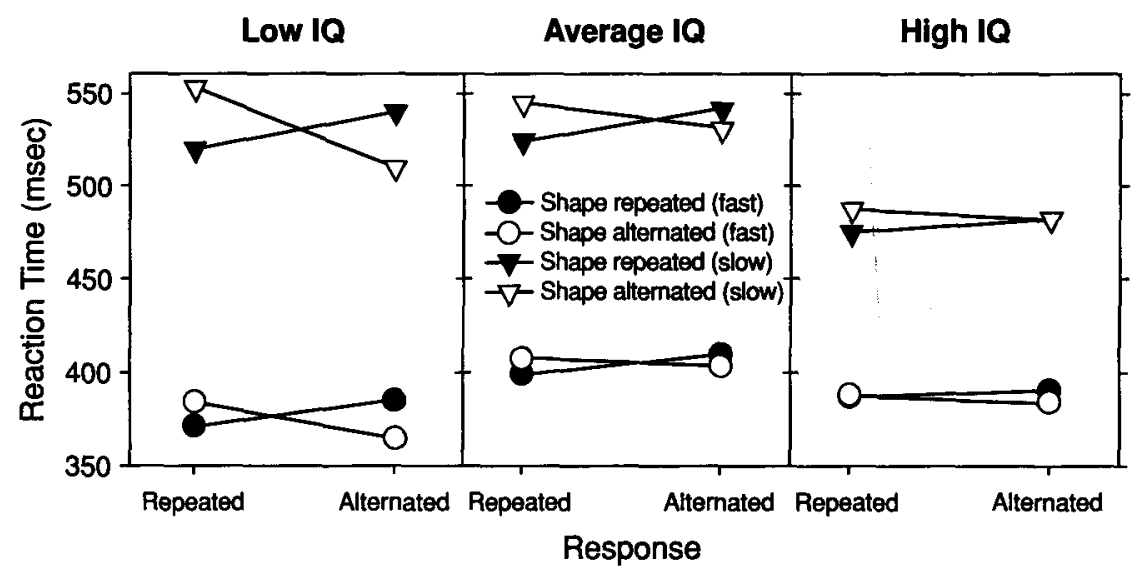

Figure 3. Mean reaction times as a function of level of IQ (low vs. average vs. high), level of relative response speed, and the repetition versus alternation of response and stimulus shape. 
are unaffected by the not-yet-completely-retrieved event file from the previous episode. Slower responses are more likely to provide a full picture - that is, to indicate the true size of partial-overlap costs. Yet, the size of these costs does not necessarily increase with the RT level, which is particularly obvious with the high-IQ group.

\section{CONCLUSIONS}

We observed that people high in fluid intelligence suffer less from the mismatch between successive pairings of a task-relevant stimulus feature and the response. In our study, shape was the only task-relevant feature, not color or location; because of the spatial responses used in our experiment, however, the latter two factors were indirectly related to the task. Given that the frontal lobe is implicated in updating relevant, but not irrelevant, information and that frontal-lobe functions are associated with intelligence, it is not surprising that fluid intelligence qualified the effect only of the directly task-related feature. Although it is clear that more research on this issue is necessary, we take our findings to be consistent with the hypothesis that fluid intelligence may facilitate feature "unbinding."

Our results suggest that high fluid intelligence is not only associated with greater mental and/or sensorimotor speed, but is also correlated with greater flexibility and efficiency in managing and updating event files-that is, in cognitive structures that interlink the codes of features belonging to the same event. Our observation fits well with the idea that high intelligence is accompanied by (or is due to) a high degree of control over the contents of working memory (Duncan et al., 2000). These contents may consist of adaptive, context-sensitive pointers (presumably in prefrontal cortex; Duncan, 2001; Miller \& Cohen, 2001) to object- and action-related feature codes in temporal and premotor cortex or elsewhere (Hommel, 2004).

It is interesting to note that intelligence was associated with stimulus-response unbinding but not with the unbinding of stimulus features. This result may have to do with the fact that only one stimulus feature was task relevant. Perhaps, if more than one stimulus feature were task relevant, IQ would modify the unbinding of those features as well. Another possibility is that the impact of intelligence is restricted, in principle, to stimulus-response links. In the already-mentioned drug studies, we found evidence that muscarinic-cholinergic agonists and antagonists have an impact on bindings involving visual features, but not on stimulus-response bindings. This finding suggests that object integration and object-action coupling are driven by different neurotransmitter systems, which would fit with the observation that partial-overlap costs associated with the (un)binding of visual features correlate with each other, but not with costs associated with stimulus-response (un)binding (Colzato, Warrens, \& Hommel, 2006). One may thus speculate that fluid intelligence and stimulus-response integration processes are related to the same neurotransmitter system, with dopamine being an obvious candidate (see, e.g., Previc, 1999).
In conclusion, our findings are consistent with the ideas that the efficiency with which people are able to manipulate and update the contents of working memory represents a fundamental aspect of human fluid intelligence (Duncan et al., 2000) and that this aspect is reflected in the ease with which they can undo previously formed episodic bindings of relevant stimulus and response features. To be sure, given the correlative nature of our study, our results should only be taken as a first, preliminary step to understanding the causal connection between feature integration and intelligence.

\section{REFERENCES}

Anokhin, A. P., Lutzenberger, W., \& Birbaumer, N. (1999). Spatiotemporal organization of brain dynamics and intelligence: An EEG study in adolescents. International Journal of Psychophysiology, 33, 259-273.

Carpenter, P. A., Just, M. A., \& Shell, P. (1990). What one intelligence test measures: $A$ theoretical account of the processing in the Raven Progressive Matrices test. Psychological Review, 97, 404-431.

Colzato, L. S., Erasmus, V., \& Hommel, B. (2004). Moderate alcohol consumption impairs feature binding in visual perception but not across perception and action. Neuroscience Letters, 360, 103-105.

Colzato, L. S., Fagioli, S., Erasmus, V., \& Hommel, B. (2005). Caffeine, but not nicotine, enhances visual feature binding. European Journal of Neuroscience, 21, 591-595.

Colzato, L. S., WARrens, M. J., \& Hommel, B. (2006). Priming and binding in and across perception and action: A correlational analysis of the internal structure of event files. Quarterly Journal of Experimental Psychology, 59, 1785-1804.

DEARY, I. J. (2000). Looking down on human intelligence: From psychometrics to the brain. Oxford: Oxford University Press.

DiAmond, A. (1990). Developmental time course in human infants and infant monkeys, and the neural bases of, inhibitory control in reaching. Annals of the New York Academy of Sciences, 608, 637-676.

DUNCAN, J. (2001). An adaptive coding model of neural function in prefrontal cortex. Nature Reviews Neuroscience, 2, 820-829.

Duncan, J., SeItz, R. J., Kolodny, J., Bor, D., Herzog, H., AHMEd, A., ET AL. (2000). A neural basis for general intelligence. Science, $289,457-460$.

Gathercole, S. E. (1999). Cognitive approaches to the development of short-term memory. Trends in Cognitive Sciences, 3, 410-418.

HASHER, L., \& ZACKS, R. T. (1988). Working memory, comprehension, and aging: A review and a new view. In G. H. Bower (Ed.), The psychology of learning and motivation (Vol. 22, pp. 193-225). San Diego: Academic Press.

HoMMEL, B. (1998). Event files: Evidence for automatic integration of stimulus response episodes. Visual Cognition, 5, 183-216.

HOMMEL, B. (2004). Event files: Feature binding in and across perception and action. Trends in Cognitive Sciences, 8, 494-500.

Hommel, B., \& Colzato, L. S. (2004). Visual attention and the temporal dynamics of feature integration. Visual Cognition, 11, 483-521.

HOMMEL, B., KRAY, J., \& LINDENBERGER, U. (2006). Sticky bindings: Feature integration from childhood to old age. Manuscript submitted for publication.

Hommel, B., Müsseler, J., Aschersleben, G., \& Prinz, W. (2001). The Theory of Event Coding (TEC): A framework for perception and action planning. Behavioral \& Brain Sciences, 24, 849-973.

JENSEN, A. R. (1993). Why is reaction time correlated with psychometric g? Current Directions in Psychological Science, 2, 53-56.

Kahneman, D., Treisman, A., \& GibBs, B. J. (1992). The reviewing of object files: Object-specific integration of information. Cognitive Psychology, 24, 175-219.

MiLLER, E. K., \& CoHEN, J. D. (2001). An integrative theory of prefrontal cortex function. Annual Review of Neuroscience, 24, 167-202.

Previc, F. H. (1999). Dopamine and the origins of human intelligence. Brain \& Cognition, 41, 299-350. 
RATCLIFF, R. (1979). Group reaction time distributions and an analysis of distribution statistics. Psychological Bulletin, 86, 446-461.

Rodriguez, R., Kallenbach, U., Singer, W., \& MUNK, M. H. (2004). Short- and long-term effects of cholinergic modulation on gamma oscillations and response synchronization in the visual cortex. Journal of Neurascience, 24, 10369-10378.

SINGER, W. (1994). The organization of sensory motor representations in the neocortex: A hypothesis based on temporal coding. In C. Umiltà \& M. Moscovitch (Eds.), Attention and performance XV: Conscious and nonconscious information processing (pp. 77-107). Cambridge, MA: MIT Press, Bradford Books.

StOET, G., \& HOMMEL, B. (1999). Action planning and the temporal binding of response codes. Journal of Experimental Psychology: Human Perception \& Performance, 25, 1625-1640.
Treisman, A. (1996). The binding problem. Current Opinion in Neurobiology, 6, 171-178.

VERNON, P. A. (ED.) (1987). Speed of information-processing and intelligence. Norwood, NJ: Ablex.

\section{NOTE}

1. In particular, we averaged the RTs of trials falling below and above the individual RT median per condition. The group means per condition presented in Figure 3 are thus different from the group mean of overall individual means in Figure 2.

(Manuscript received December 11, 2005; revision accepted for publication April 17, 2006.) 